\title{
Segregation of default mode from frontoparietal system protects against smoking lapse
}

Lydon-Staley, D.M. ${ }^{1,2}$, MacLean, R.R. ${ }^{3,4}$, Falk, E.B. ${ }^{2,5,6}$, Bassett, D.S. ${ }^{1,7,8,9,10,11}$, \& Wilson, S.J. ${ }^{12 *}$

${ }^{1}$ Department of Bioengineering, School of Engineering and Applied Science, University of

Pennsylvania, Philadelphia, PA

${ }^{2}$ Annenberg School for Communication, University of Pennsylvania, Philadelphia, PA

${ }^{3}$ VA Connecticut Healthcare System, West Haven, CT

${ }^{4}$ Yale University School of Medicine, New Haven, CT

${ }^{5}$ Department of Psychology, University of Pennsylvania, Philadelphia, PA

${ }^{6}$ Wharton Marketing Department, University of Pennsylvania, Philadelphia, PA

${ }^{7}$ Department of Electrical \& Systems Engineering, School of Engineering \& Applied Science,

University of Pennsylvania, Philadelphia, PA

${ }^{8}$ Department of Neurology, Perelman School of Medicine, University of Pennsylvania,

Philadelphia, PA

${ }^{9}$ Department of Psychiatry, Perelman School of Medicine, University of Pennsylvania, Philadelphia, PA

${ }^{10}$ Department of Physics \& Astronomy, College of Arts \& Sciences, University of Pennsylvania, Philadelphia, PA

${ }^{11}$ The Santa Fe Institute, Sante Fe, NM

${ }^{12}$ Department of Psychology, The Pennsylvania State University, University Park, PA

*Corresponding author: Stephen Wilson, 311 Moore Building, The Pennsylvania State

University, University Park, PA 16802 USA. Email: sjw42@psu.edu. Ph: 814-865-6219. 


\begin{abstract}
Background. Quitting smoking is notoriously difficult. Models of nicotine dependence posit that deficits in cognitive control contribute to failures to maintain smoking abstinence during smoking cessation attempts. We examine the role for large-scale functional brain systems associated with cognitive control in smoking lapse. Methods. We use data from 70, five-minute functional magnetic resonance imaging (fMRI) scans in 17 daily smokers ( 5 female) undergoing a smoking lapse paradigm after 12 hours of smoking abstinence. Results. Cox regression results indicate that decreased segregation of the default mode system from the frontoparietal system undermine the ability to resist smoking. Conclusions. Results lend support to the hypothesis that large-scale functional brain systems associated with cognitive control are implicated in smoking lapse behavior and point to the importance of cognitive control as a mechanism underlying smoking relapse.
\end{abstract}

Keywords: nicotine; tobacco; fMRI; lapse; connectivity 


\section{INTRODUCTION}

Quitting smoking is notoriously difficult, with the majority of cessation attempts ending in relapse (i.e., return to regular smoking) (1). Models of nicotine dependence posit that deficits in cognitive control contribute to continued smoking after a quit attempt in two key ways. A reduced ability to override impulses to smoke can bias decisions towards smoking over alternative reinforcers $(2,3)$ and can promote a return to smoking in order to ameliorate abstinence-related cognitive control deficits (4). In line with these proposals, smokers exhibit impaired inhibitory control and working memory relative to non-smokers $(5,6)$; smokers show impaired cognitive control performance during smoking abstinence relative to smoking satiety $(7,8)$; and poorer performance on working memory tasks and inhibitory control tasks is associated with more rapid smoking resumption following smoking abstinence $(9,10)$. Here, we extend research on the role of cognitive control in smoking cessation by examining how functional brain systems associated with cognitive control are correlated with smoking lapse.

One of the best predictors of relapse (11) following a smoking cessation attempt is experiencing a lapse (i.e., any smoking after initial cession) (12). Although lapses can represent just a single puff on a cigarette (13), the majority of participants who lapse go on to relapse and resume regular smoking $(14,15)$. The first lapse during a cessation attempt, then, often represents a gateway towards relapse $(16,17)$. Findings that cognitive control is associated with relapse provide initial evidence that limitations in cognitive control are implicated in smoking lapses $(10,18)$.

Yet, the nature of lapses, as defined by discrete moments embedded within cessation attempts, challenges the identification of their precipitants. A second challenge for studying smoking lapses is that the precipitants of lapses may themselves change from moment to 
moment. Indeed, in the case of cognitive control processes such as working memory, inhibitory control, and sustained attention, substantial day-to-day (19) and even moment-to-moment $(20,21)$ fluctuations in performance have been observed. Within-person fluctuations have also been observed in the functional brain organization of systems involved in cognitive control $(22,23)$. Observations of fluctuations in cognitive control and cognitive control-relevant functional brain systems encourage a consideration of functional brain systems in the moments immediately preceding lapses given that the status of processes (e.g., negative affect) in moments more proximal to lapses are more predictive of smoking lapses than data collected further back in time (e.g., hours before lapse versus day before lapse) (24).

Laboratory smoking lapse paradigms represent an efficient and cost-effective way to overcome the difficulty of isolating lapse behaviors as they occur (25). In an increasingly used lapse paradigm, smokers are exposed to known precipitants of smoking relapse behavior, including nicotine deprivation, alcohol, and stress (26-28). Smokers are then given the option of beginning tobacco self-administration or delaying self-administration by 5 -minute increments for up to 50 minutes in exchange for monetary reinforcement. The delay period models smokers' ability to resist smoking, with shorter relative to longer times to lapse reflective of lower abilities to resist smoking. This lapse paradigm demonstrates validity, showing sensitivity to the effects of medications with known clinical efficacy for smoking cessation (29) and, to date, has supported the role of alcohol, stress, nicotine and food deprivation, exposure to smoking environment cues, and the devaluation of monetary rewards in facilitating lapse behavior in the laboratory $(26,28,30-33)$.

In the present study, we overcome the challenges associated with identifying the role of cognitive control-relevant functional brain systems in smoking lapses by observing participants 
as they engage in a laboratory smoking lapse paradigm, accompanied by functional Magnetic Resonance Imaging (fMRI). Our goal is to provide insight into the role of cognitive controlrelevant functional brain systems in efforts to resist the urge to smoke. We focus on interactions between two large-scale functional brain systems that are known to support cognitive control. The frontoparietal system is comprised of regions with roles in response selection (34), response suppression (35), working memory (36), and attentional control (37), processes shown to be relevant for changes in smoking behavior $(9,10,18)$. The default mode system is characterized by a tendency to deactivate during many cognitive tasks and to activate at rest, as well as during self-referential and social tasks $(38,39)$. Evidence suggests that the integrity of functional connectivity of the default mode and frontoparietal systems, as well as their interactions, is fundamental to cognitive control. In particular, greater strength of connectivity among the default mode and frontoparietal systems, indicating reduced segregation of activity between the two systems, is associated with poorer working memory and inhibitory control performance (40-42). Given the association between cognitive control and the segregation of the default mode from the frontoparietal system, we hypothesized that greater segregation of the default mode from the frontoparietal system would protect against lapses during a smoking lapse paradigm. Notably, by taking continuous fMRI measurements during the course of the lapse task, we also capture potentially time-varying changes in functional connectivity of the systems theorized to be associated with lapse behavior.

\section{METHODS AND MATERIALS}

\section{Participants}


Participants were 20 individuals ( 8 female) recruited using newspaper, radio, and internet advertisements, and flyers posted in the community. To be eligible for the study, individuals were required to be right handed, to be between the ages of 18 and 45 years, to report that they smoked at least 10 cigarettes per day for the past 24 months, to indicate that they were not currently planning to quit smoking or actively pursuing any form of smoking cessation treatment, and to have a baseline expired-air carbon monoxide $(\mathrm{CO})$ level greater than 10 parts per million (ppm); the latter criterion was chosen in order to verify smoking status (BreathCo, Vitalograph, Lenexa, Kansas). Individuals were excluded if they reported any of the following: current heavy use of illicit substances (defined as illicit drug use on 10 or more days in the past 30 days), current use of prescription medications that have been found to affect blood flow responses in the brain, current psychiatric diagnoses, chronic cardiovascular or respiratory problems, and/or any contraindications for magnetic resonance imaging (MRI). All procedures were approved by the Pennsylvania State University Institutional Review Board, and written informed consent was obtained from all participants.

\section{Procedures}

Demographic and smoking-related variables were collected at an initial baseline session. Participants then completed an fMRI session on a subsequent day. They were instructed to abstain from smoking and from using any nicotine-containing products for at least 12 hours prior to the scan session. Upon arriving for the fMRI experiment, participants reported the last time they smoked a cigarette and a CO sample was obtained to verify compliance with these instructions; compliance was defined as $<8$ ppm or $\geq 50 \%$ reduction from their baseline CO level. Participants then completed the following surveys to assess affective state, nicotine withdrawal symptoms, and the urge to smoke: the Positive and Negative Affect Schedule (43), 
the Wisconsin Smoking Withdrawal Scale (44), and the Questionnaire of Smoking Urges-Brief $(45)$.

After completion of the surveys and before being placed in the scanner, participants were given instructions for an fMRI reward task not reported on here. Following the acquisition of anatomical data, participants then completed a 3.5-minute resting baseline scan and six runs of the reward task, each lasting approximately 5 minutes. Next, participants performed an fMRI task modeling smoking lapse behavior adapted from prior behavioral research (27), which is the focus of the current study.

At the beginning of the fMRI smoking lapse task, participants were informed that they would be given the opportunity to smoke immediately after being removed from the scanner, but that they would be given the chance to earn extra money by delaying their removal to complete additional scans. Specifically, they were told that they could choose to remain in the scanner for up to 50 additional minutes, earning $\$ 1$ for every five minutes that they remained in the scanner (i.e., up to $\$ 10$ total), and that they would be asked to indicate via button press whether or not they would like to remain in the scanner before each five-minute scan began. After receiving instructions, participants provided visual analog scale ratings of their affect (from "unpleasant" to "pleasant"), their level of arousal (from "sleepy" to "aroused/activated"), and their urge to smoke (from "no urge at all" to "strongest urge ever"), with each scored on a 0-100 range.

Participants then completed the following sequence for each of up to 10 five-minute runs. First, participants pushed one of two buttons to signify whether they would like to start the subsequent 5-minute run of the smoking lapse task or be removed from the scanner. Next, those who elected to remain in the scanner completed a 5-minute run of the task, during which they were asked to relax and remain as still as possible with their eyes open. (If the participant instead 
chose to be removed from the scanner, they were taken out of the MRI at that point and did not complete any additional runs of the task.) Finally, immediately after the run ended, participants used visual analog scales to rate their affect, level of arousal, and urge to smoke, as well as to rate how much they were trying to change or resist their urge to smoke during the previous run (from "not at all" to "very much"). Participants were given the opportunity to smoke after being removed from the scanner and were paid the money that they earned during the smoking lapse task in cash at the conclusion of the experimental session.

\section{Data Preparation}

A summary of our preparation and analysis of the functional imaging data from the smoking lapse task is as follows: we preprocessed and denoised the BOLD time series, after which we created an association matrix representing the functional connectivity among regions of the brain for each participant and each 5-minute block. We then quantified the segregation of the default mode from the frontoparietal system for each association matrix. We provide additional detail below.

Data acquisition. Scanning was conducted at the Penn State Social, Life, and Engineering Sciences Imaging Center using a 3-Tesla Siemens Trio scanner (Siemens Corporation, NY). Prior to functional scanning, a high-resolution T1-weighted (T1w) anatomical image was acquired (256x256 matrix; FOV=256 mm²; 160 1-mm sagittal slices). During functional scanning, 34-slice oblique-axial functional images (3x3x3 mm voxels) were acquired using a standard echo-planar imaging pulse sequence $[\mathrm{TR}=2000 \mathrm{~ms}, \mathrm{TE}=25 \mathrm{~ms}, \mathrm{FOV}=192 \mathrm{~mm}$, flip angle $\left.=80^{\circ}\right]$.

Data preprocessing. Initial preprocessing of the brain imaging data was performed using fMRIPrep 1.4.1rc1 (46), which is based on Nipype 1.2.0 $(47,48)$. A detailed overview of the 
steps taken is provided in the supplement. We then denoised the fMRI data with a protocol based on studies that evaluated the performance of a wide variety of denoising pipelines in mitigating motion artifact in studies of BOLD functional connectivity $(49,50)$ using the publicly available eXtensible Connectivity Pipeline (XCP) software (51). Six head motion regressors and three matter regressors (global signal, white matter, and cerebrospinal fluid), as well as their derivatives, quadratic terms, and the squares of their derivatives (36 regressors in total) were regressed from the time series. We also conducted despiking - identifying outliers in the intensity of each voxel's detrended BOLD time series and interpolating over these outliers.

Creating an association matrix. Using the preprocessed and denoised BOLD fMRI data, we created an association matrix representing the strength of functional connectivity between pairs of brain regions. We defined regions of the default mode and frontoparietal systems on a commonly applied parcellation scheme (52), the coordinates of which can be found in the Supporting Information. For each region, we extracted a time series of the BOLD signal separately for each individual. All regions were modeled as $10 \mathrm{~mm}$ diameter spheres around the center coordinates. The extracted time series were the average time series for all voxels within the sphere. The spherical regions represented nodes in functional connectivity networks. Pairwise Pearson correlation coefficients between node time series were used as network edge weights. Similar to previous functional connectivity studies (e.g., 53,54), negative correlations were set to 0 to eliminate potential misinterpretation of negative edge weights.

Default mode and frontoparietal system segregation measure. We calculated the strength of default mode system segregation from the frontoparietal system as follows (55): Default Mode System Segregation $=\frac{\bar{z}_{w}-\bar{z}_{b}}{\bar{z}_{w}}$, 
where $\bar{z}_{w}$ is the mean connectivity strength of edges between all pairs of nodes in the default mode system and $\bar{z}_{b}$ is the mean connectivity strength of edges between all pairs of nodes that spanned the default mode and the frontoparietal systems. Higher values of the default mode system segregation indicate greater segregation of the default mode system from the frontoparietal system.

\section{Data Analysis}

We hypothesized that segregation of the default mode from the frontoparietal system would be protective against deciding to leave the scanner in order to smoke a cigarette rather than remaining in the scanner in order to earn a monetary incentive. We adopted a survival analysis framework, a framework in which the outcome variable is the timing of an event (56), to test this hypothesis. Participants who experience an event during the observational period experience a "death", whereas participants not experiencing an event "survive" (the terminology comes from survival analysis' legacy in epidemiology). In the current analyses, the relevant event is the decision to leave the scanner. In the present study, there were 9 event times, or moments at which a participant could decide to leave the scanner during the smoking lapse task (i.e., at the end of blocks 1 though 9). Of the 20 participants enrolled in the study, three did not choose to leave the scanner at any point during the 10 blocks. Survival analysis was developed in part to handle right-censored cases and, as such, these three right-censored cases are readily accommodated (57). Three participants decided to smoke rather than to begin the smoking lapse task. These left censored cases, in which the event occurred prior to or coincident with the start of the observation period, were not included in the analysis as they provided no fMRI data for the smoking lapse task. 
A participant's likelihood of survival may be associated with different types of predictors, both time-invariant (e.g., age) and time-varying (e.g., segregation of the default mode from the frontoparietal system within each scanning block). We estimate the survival function, reflecting the cumulative loss of all participants in the sample. Formally, the survival function is expressed as

$S(t)=\operatorname{Pr}(T>t)$

and gives the probability that a participant will survive past time $t$. We also estimate the hazard rate, which is the risk of experiencing the event of interest given that the participant has survived up to a specific time, and we determine whether the function differs systematically in relation to predictor variables. We use a Cox regression model to examine how the hazard rate is related to default mode segregation from the frontoparietal system during each scanning block of the smoking lapse task. We fit a cox regression model specified as

$h_{i}(t)=h_{0}(t) \exp \left(\beta_{1}\right.$ Age $_{i}+\beta_{2}$ Segregation $\left._{i}(t)\right)$,

where the hazard of deciding to leave the scanner at time $t$ depends on the product of the baseline hazard $h_{0}(t)$ and an exponentiated linear function of $q$ predictors that may be time-invariant or time-varying. We include the time-invariant predictor of age, $\beta_{1}$, and the time-varying predictor of default mode segregation from the frontoparietal system during each scanning block directly preceding the decision, $\beta_{2}$, the value of which varies from scanning block to block.

Of greatest interest was the test of whether the parameter $\beta_{2}$ was different than 0 (i.e., that there is an association between default mode-frontoparietal system segregation and the hazard of choosing to leave the scanner to smoke a cigarette). Parameters were transformed into a more easily interpreted hazard ratio metric $(\mathrm{HR}=\exp [\beta])$, which can be interpreted as the change in the risk of leaving the scanner if the parameter in question rises by one unit: $\mathrm{HR}=1.00$ 
indicates no association between the predictor and outcome variable, $\mathrm{HR}>1$ indicates higher hazard of event occurrence for higher values of the predictor, and $\mathrm{HR}<1$ indicates lower hazard of even occurrence for higher values of the predictor (58). HRs can also be interpreted as percent change in hazard as $100 \times$ [HR-1]. We included age as a time-invariant covariate because previous work indicates that default mode and frontoparietal system segregation decreases with age (e.g.,59).

We fit the model using PROC PHREG (60) by implementing the counting process style of input (61). The discrete nature of event quantification (block by block) resulted in tied times during which participants decided to leave the scanner. We used the "tie = exact" option in SAS Proc PHREG to accommodate these tied events (62).

\section{RESULTS}

\section{Participant characteristics and descriptive statistics}

As detailed above, 17 participants ( 5 female) provided data for the survival analysis. The mean age of these participants was 24.41 years $(S D=6.90)$. The self-identified racial composition of the usable sample was as follows: $82 \%$ White, $6 \%$ Asian, and $12 \%$ unreported. Participants reported smoking an average of $13.41(S D=3.62)$ cigarettes per day and had a baseline CO level of $19.53 \mathrm{ppm}(S D=7.19)$. Additional characterization of the sample may be found in Table S1.

We provide descriptive statistics of key model variables in Table 1. Figure 1 shows the survival times for each participant (presence of square on horizontal lines) grouped by scanning block, in addition to the value of default mode frontoparietal system segregation within each block for each participant. Right censored cases $(n=3)$ do not have Xs at the end of their rows to indicate that the event of interest (smoking lapse) was not observed in these participants. 


\section{Segregation of the default mode from the frontoparietal system protects against lapse}

The baseline survival function estimated from an unconditional (baseline hazard) model is shown in Figure 2. We then added age and the default mode frontoparietal system segregation variable to the model. Goodness of model fit was tested using a likelihood ratio test that compared the fit of the model with age and default mode frontoparietal system segregation as predictors relative to the unconditional model. The likelihood ratio test was significant, $\chi^{2}(2)=7.02, p=0.03$, indicating that the model with age and default mode frontoparietal system segregation fit the data better than the unconditional model.

Results of the model (Table 2) indicate that the extent of default mode frontoparietal system segregation in the scanning block immediately preceding the decision to stay or leave the scanner was associated with the choice to leave the scanner in order to smoke a cigarette, $\beta_{2}=-$ $5.84, p=0.04$. As hypothesized, with one unit increase in the segregation variable, participants were 0.003 times $(\mathrm{HR}=0.003)$ as likely, or 99.7\% less likely (percent change $=100 \mathrm{x}[0.003-$ $1.00]=-99.7 \%$ ), to choose to leave the scanner in order to smoke a cigarette.

\section{Results are robust to participant motion}

Follow-up analyses indicate that the association between default mode frontoparietal system segregation and the choice to leave the scanner was robust to including participant motion (Table S2). Examining the independent associations between the components that make up the default mode frontoparietal system segregation variable revealed that connectivity between the default mode and frontoparietal systems was the biggest driver of the observed association (Table 3), relative to within-system functional connectivity of the default mode system and the frontoparietal system.

No association is observed between self-reports and smoking lapse 
Time-varying self-reports of affect, arousal, and urge to smoke prior to each scan block, and post scan block self-ratings of how much participants were trying to change or resist their urge to smoke during the previous run, were not significantly associated with the choice to leave the scanner in order to smoke (Table S4). Repeated measure correlations between the default mode frontoparietal system segregation measure and the self-report scales indicated no significant associations between segregation and urge to smoke, $r(52)=0.09, p=0.54$, arousal, $r(52)=0.09, p=0.52$, or efforts to resist smoking urges, $r(52)=-0.07, p=0.61$. However, affect was significantly correlated with segregation, $r(52)=0.33, p=0.02$, such that greater segregation of the default mode and frontoparietal systems was associated with more positive affect prior to the scan block.

\section{DISCUSSION}

One of the best predictors of smoking relapse is the experience of a lapse during a cessation attempt $(14,16)$. To provide insight into the precipitants of smoking lapses, we examined the association between cognitive control-relevant functional brain systems and smoking lapse behavior. In line with our hypothesis, decreased segregation of the default mode system from the frontoparietal system undermined the ability to resist smoking in a sample of daily smokers who were deprived of nicotine for over 12 hours.

The current study extends a laboratory paradigm modeling smoking lapse behavior (27) into the neuroimaging setting. Capturing BOLD fMRI during an attempt to resist smoking to earn a monetary incentive allowed us to test the role for large-scale functional brain networks associated with cognitive control in prompting lapse behavior. Our focus on connectivity among the default mode and frontoparietal systems reflects the importance of these systems in cognitive 
control abilities, with findings that segregation of these systems from one another supports accurate cognitive control performance (40-42). In the context of cigarette-smoking specifically, improvements in cognitive withdrawal symptoms after nicotine replacement are associated with increased inverse coupling between default mode and frontoparietal systems (63). Our findings are consistent with behavioral studies indicating that poorer cognitive control task performance is associated with more rapid smoking resumption following smoking abstinence $(9,10)$, and further build upon them by considering brain dynamics in real time during decisions to resist smoking.

Interestingly, self-ratings of affect, arousal, and urge to smoke prior to scan blocks and post-scan block ratings of how much participants resisted the urge to smoke were not significantly associated with decisions to leave the scanner in order to smoke a cigarette. These findings add to a body of literature indicating the promise of neural activity in predicting smoking-related behaviors (64-68). For example, existing work has observed associations between BOLD activity in the left dorsolateral prefrontal cortex and posterior cingulate during an N-back working memory task and the ability to remain abstinent during a 7-day quit attempt (69). With these findings, neuroimaging is emerging as a tool to predict behavior, providing an alternative to efforts to predict future behavior through self-reports that may contain biases stemming from social desirability effects (70) or that may fail to predict behavior due to a lack of conscious access to factors implicated in behavior (71).

Although self-ratings were not associated with lapse behavior, a correlation was observed between self-ratings of affect and default mode frontoparietal system segregation. More positive affect was reported prior to scans during which segregation between the default mode and frontoparietal systems was greater than usual. Positive affect has been theorized to inhibit 
craving by facilitating self-regulation (72) and, consistent with this perspective, high positive affect is associated with reduced cravings during tobacco, alcohol, and opioid withdrawal $(73,74)$. Results of the present study, coupled with findings that positive mood is associated with increased flexibility in large-scale brain networks (75), are consistent with the perspective that positive mood facilitates self-regulation via modulating functional connectivity associated with cognitive control. Notably, however, functional connectivity predicted lapse behavior above and beyond self-rating of affect (Table S5) suggesting that while positive affect may modulate the extent of segregation between the default mode and frontoparietal systems, it is not sufficient to predict lapse behavior on its own.

\section{Conclusions}

In summary, segregation of the default mode system from the frontoparietal system was associated with a reduced risk of lapsing during a laboratory smoking lapse task. Results lend support to the hypothesis that large-scale functional brain systems associated with cognitive control are implicated in smoking lapse behavior and point to the importance of cognitive control as a mechanism underlying smoking relapse. 


\section{Funding}

S.J.W. acknowledges support from the National Institute on Drug Abuse (R01DA041438 and R21DA045853). D.S.B. and D.M.L. acknowledge support from the John D. and Catherine T. MacArthur Foundation, the Alfred P. Sloan Foundation, the ISI Foundation, the Paul Allen Foundation, the Army Research Laboratory (W911NF-10-2-0022), the Army Research Office (Bassett-W911NF-14-1-0679, Grafton-W911NF-16-1-0474, DCIST- W911NF-17-2-0181), the Office of Naval Research, the National Institute of Mental Health (2-R01-DC-009209-11, R01 MH112847, R01-MH107235, R21-M MH-106799), the National Institute of Child Health and Human Development (1R01HD086888-01), National Institute of Neurological Disorders and Stroke (R01 NS099348), and the National Science Foundation (BCS-1441502, BCS-1430087, NSF PHY-1554488 and BCS-1631550). D.M.L. acknowledges support from the National Institute on Drug Abuse (1K01DA047417-01A1). D.M.L, D.S.B, and E.B.F acknowledge support from the Army Research Office (W911NF-18-1-0244). The content is solely the responsibility of the authors and does not necessarily represent the official views of any of the funding agencies. 
Disclosure of Biomedical Financial Interests and Potential Conflicts of Interest

The authors have no conflicts of interest to disclose. 


\section{Citation diversity statement}

Recent work in neuroscience and other fields has identified a bias in citation practices such that papers from women and other minorities are under-cited relative to the number of such papers in the field (76-81). Here we sought to proactively consider choosing references that reflect the diversity of the field in thought, form of contribution, gender, and other factors. We used automatic classification of gender based on the first names of the first and last authors $(79,82)$, with possible combinations including male/male, male/female, female/male, and female/female. Excluding self-citations to the first and last authors of our current paper, the references contain $38.10 \%$ male/male, $15.87 \%$ male/female, $23.81 \%$ female/male, $17.46 \%$ female/female, and 4.76\% unknown categorization. We look forward to future work that could help us to better understand how to support equitable practices in science. 


\section{References}

1. Chaiton M., Diemert L., Cohen JE, Bondy SJ, Selby P, Philipneri A, and Schwartz, R. (2016): Estimating the number of quit attempts it takes to quit smoking successfully in a longitudinal cohort of smokers. BMJ Open 6:e011045.

2. Bechara A. (2005): Decision making, impulse control and loss of willpower to resist drugs: a neurocognitive perspective. Nat Neurosci 8:1458-1463.

3. Goldstein RZ, and Volkow ND (2002): Drug addiction and its underlying neurobiological basis: neuroimaging evidence for the involvement of the frontal cortex. Am J Psychiatry 159:1642-1652.

4. Evans DE, To CN, and Ashare RL (2018): The role of cognitive control in the self-regulation and reinforcement of smoking behavior. Nicotine Tob Res 21: 747-754.

5. Greenstein JE, and Kassel JD (2009): The effects of smoking and smoking abstinence on verbal and visuospatial working memory capacity. Exp Clin Psychopharm 17: 78-90.

6. Spinella M (2002): Correlations between orbitofrontal dysfunction and tobacco smoking. Addict Biol 7: 381-384.

7. Dawkins L, Powell JH, West R, Powell J, and Pickering A (2007): A double-blind placebocontrolled experimental study of nicotine: II-Effects on response inhibition and executive functioning. Psychopharmacology 190: 457-467.

8. Geier CF, Roberts N, and Lydon-Staley DM (2018): The Effects of Smoking Abstinence on Incentivized Spatial Working Memory. Subst Use Misuse 53: 86-93.

9. Patterson F, Jepson C, Loughead J, Perkins K, Strasser AA, Siegel S, et al. (2010): Working memory deficits predict short-term smoking resumption following brief abstinence. Drug Alcohol Depend 106: 61-64. 
10. Powell J, Dawkins L, West R, Powell J, and Pickering A (2010): Relapse to smoking during unaided cessation: clinical, cognitive and motivational predictors. Psychopharmacology 212: 537-549.

11. Shiffman S (2006): Reflections on smoking relapse research. Drug Alcohol Rev 25: 15-20.

12. Kirchner TR, Shiffman S, and Wileyto EP (2012): Relapse dynamics during smoking cessation: recurrent abstinence violation effects and lapse-relapse progression. J Abnorm 121: $187-197$.

13. Brandon TH, Tiffany ST, Obremski KM, and Baker TB (1990): Postcessation cigarette use: The process of relapse. Addict Behav 15: 105-114.

14. Garvey AJ, Bliss RE, Hitchcock JL, Heinold JW, and Rosner B (1992): Predictors of smoking relapse among self-quitters: a report from the Normative Aging Study. Addict Behav 17: 367-377.

15. Shiffman S, Paty JA, Gnys M, Kassel JA, and Hickcox M (1996): First lapses to smoking: within-subjects analysis of real-time reports. J Consult Clin Psychol 64: 366-379.

16. Ashare RL, Wileyto EP, Perkins KA, and Schnoll RA (2013): The first seven days of a quit attempt predicts relapse: Validation of a measure for screening medications for nicotine dependence. $J$ Addict Med 7: 249-254.

17. Juliano LM, Donny EC, Houtsmuller EJ, and Stitzer ML (2006): Experimental evidence for a causal relationship between smoking lapse and relapse. J Abnorm 115: 166-73.

18. Krishnan-Sarin S, Reynolds B, Duhig AM, Smith A, Liss T, McFetridge A, et al. (2007). Behavioral impulsivity predicts treatment outcome in a smoking cessation program for adolescent smokers. Drug Alcohol Depend 88: 79-82. 
19. Brose A, Schmiedek F, Lövdén M, and Lindenberger U (2012): Daily variability in working memory is coupled with negative affect: the role of attention and motivation. Emotion 12: 605-617.

20. Esterman M, Rosenberg MD, and Noonan SK (2014): Intrinsic fluctuations in sustained attention and distractor processing. $J$ Neurosci 34:1724-1730.

21. Jones A, Tiplady B, Houben K, Nederkoorn C, and Field, M. (2018): Do daily fluctuations in inhibitory control predict alcohol consumption? An ecological momentary assessment study. Psychopharmacology 235: 1487-1496.

22. Braun U, Schäfer A, Walter H, Erk S, Romanczuk-Seiferth N, Haddad L, et al. (2015):

Dynamic reconfiguration of frontal brain networks during executive cognition in humans. Proc Natl Acad Sci 112: 11678-11683.

23. Chen T, Cai W, Ryali S, Supekar K, and Menon V (2016): Distinct global brain dynamics and spatiotemporal organization of the salience network. PLoS Biol 14.

24. Shiffman S, and Waters AJ (2004): Negative affect and smoking lapses: a prospective analysis. J Consult Clin Psychol 72: 192-201.

25. Lerman C, LeSage MG, Perkins KA, O'Malley SS, Siegel SJ, Benowitz NL, and Corrigall WA (2007): Translational research in medication development for nicotine dependence. Nat Rev Drug Discov 6: 746-762.

26. McKee SA, Sinha R, Weinberger AH, Sofuoglu M, Harrison EL, Lavery M, and Wanzer J (2011): Stress decreases the ability to resist smoking and potentiates smoking intensity and reward. J Psychopharmacology 25:490-502.

27. McKee SA (2009): Developing human laboratory models of smoking lapse behavior for medication screening. Addict Biol 14:99-107. 
28. McKee SA, Krishnan-Sarin S, Shi J, Mase T, and O’Malley SS (2006): Modeling the effect of alcohol on smoking lapse behavior. Psychopharmacology 189: 201-210.

29. McKee SA, Weinberger AH, Shi J, Tetrault J, and Coppola S (2012): Developing and validating a human laboratory model to screen medications for smoking cessation. Nicotine Tob Res 14: 1362-1371.

30. Leeman RF, O’Malley SS, White MA, and McKee SA (2010): Nicotine and food deprivation decrease the ability to resist smoking. Psychopharmacology 212: 25-32.

31. Kahler CW, Metrik J, Spillane NS, Day A, Leventhal AM, McKee SA, et al. (2014): Acute effects of low and high dose alcohol on smoking lapse behavior in a laboratory analogue task. Psychopharmacology 231: 4649-4657.

32. Stevenson JG, Oliver JA, Hallyburton MB, Sweitzer MM, Conklin CA, and McClernon FJ (2017): Smoking environment cues reduce ability to resist smoking as measured by a delay to smoking task. Addict Behav 67:49-52.

33. Wilson SJ, Delgado MR, McKee SA, Grigson PS, MacLean RR, Nichols TT, and Henry SL (2014): Weak ventral striatal responses to monetary outcomes predict an unwillingness to resist cigarette smoking. Cogn Affect Behav Neurosci 14: 1196-1207.

34. Jiang Y, and Kanwisher N (2003): Common neural substrates for response selection across modalities and mapping paradigms. J Cogn Neurosci 15: 1080-1094.

35. Ridderinkhof KR, Van Den Wildenberg WP, Segalowitz SJ, and Carter CS (2004):

Neurocognitive mechanisms of cognitive control: the role of prefrontal cortex in action selection, response inhibition, performance monitoring, and reward-based learning. Brain Cognition 56: 129-140. 
36. Mars RB, and Grol MJ (2007): Dorsolateral prefrontal cortex, working memory, and prospective coding for action. $J$ Neurosci 27: 1801-1802.

37. Shomstein S, Kravitz DJ, and Behrmann M (2012): Attentional control: Temporal relationships within the fronto-parietal network. Neuropsychologia 50: 1202-1210.

38. Mazoyer B, Zago L, Mellet E, Bricogne S, Etard O, Houdé O, et al. (2001): Cortical networks for working memory and executive functions sustain the conscious resting state in man. Brain Res Bull 54: 287-298.

39. Shulman GL, Fiez JA, Corbetta M, Buckner R, Miezin FM, Raichle ME, and Petersen SE (1997): Common blood flow changes across visual tasks: II. Decreases in cerebral cortex. J Cogn Neurosci 9: 648-663.

40. Hampson M, Driesen N, Roth JK, Gore JC, and Constable RT (2010): Functional connectivity between task-positive and task-negative brain areas and its relation to working memory performance. Magn Reson Imaging 28: 1051-1057.

41. Kelly AC, Uddin LQ, Biswal BB, Castellanos FX, and Milham MP (2008): Competition between functional brain networks mediates behavioral variability. NeuroImage 39:527537.

42. Murphy AC, Bertolero MA, Papadopoulos L, Lydon-Staley DM, and Bassett DS (2019): Multiscale and multimodal network dynamics underpinning working memory. arXiv preprint arXiv:1901.06552.

43. Watson D, Clark LA, and Tellegen A (1988): Development and validation of brief measures of positive and negative affect: the PANAS scales. J Pers Soc Psychol 54: 1063-1070. 
44. Welsch, S. K., Smith, S. S., Wetter, D. W., Jorenby, D. E., Fiore, M. C., \& Baker, T. B. (1999). Development and validation of the Wisconsin Smoking Withdrawal Scale. Experimental and Clinical Psychopharmacology, 7, 354-361.

45. Cox LS, Tiffany ST, and Christen AG (2001): Evaluation of the brief questionnaire of smoking urges (QSU-brief) in laboratory and clinical settings. Nicotine Tob Res 3: 7-16.

46. Esteban O, Markiewicz CJ, Blair RW, Moodie CA, Isik AI, Erramuzpe A, et al. (2019): fMRIPrep: a robust preprocessing pipeline for functional MRI. Nat Methods 16: 111-116.

47. Gorgolewski K, Burns CD, Madison C, Clark D, Halchenko YO, Waskom ML, and Ghosh SS (2011): Nipype: a flexible, lightweight and extensible neuroimaging data processing framework in python. Front Neuroinform 5: 13.

48. Gorgolewski KJ, Alfaro-Almagro F, Auer T, Bellec P, Capotă M, Chakravarty MM, et al. (2017): BIDS apps: Improving ease of use, accessibility, and reproducibility of neuroimaging data analysis methods. PLoS Comput Biol 13: e1005209.

49. Ciric R, Wolf DH, Power JD, Roalf DR, Baum GL, Ruparel K, et al. (2017): Benchmarking of participant-level confound regression strategies for the control of motion artifact in studies of functional connectivity. NeuroImage 154: 174-187.

50. Lydon-Staley DM, Ciric R, Satterthwaite TD, and Bassett DS (2019): Evaluation of confound regression strategies for the mitigation of micromovement artifact in studies of dynamic resting-state functional connectivity and multilayer network modularity. Network Neurosci 3: 427-454.

51. Ciric R, Rosen AF, Erus G, Cieslak M, Adebimpe A, Cook PA, et al. (2018): Mitigating head motion artifact in functional connectivity MRI. Nat Protoc 13: 2801-2826. 
52. Power JD, Cohen AL, Nelson SM, Wig GS, Barnes KA, Church JA, et al. (2011): Functional network organization of the human brain. Neuron 72: 665-678.

53. Chan MY, Park DC, Savalia NK, Petersen SE, and Wig GS (2014): Decreased segregation of brain systems across the healthy adult lifespan. Proc Natl Acad Sci 111: E4997-E5006.

54. Grady C, Sarraf S, Saverino C, and Campbell K (2016): Age differences in the functional interactions among the default, frontoparietal control, and dorsal attention networks. Neurobiol Aging 41: 159-172.

55. Cohen JR, and D'Esposito M (2016): The segregation and integration of distinct brain networks and their relationship to cognition. $J$ Neurosci 36: 12083-12094.

56. Allison PD (1984): Event history analysis. Regression for longitudinal event data. Newbury Park, CA: Sage.

57. Singer JD, and Willett JB (2003): Applied longitudinal data analysis: Modeling change and event occurrence. Oxford: Oxford University Press.

58. Mills M (2010): Introducing survival and event history analysis. London, UK: Sage.

59. Ng KK, Lo JC, Lim JK, Chee MW, and Zhou J (2016): Reduced functional segregation between the default mode network and the executive control network in healthy older adults: a longitudinal study. NeuroImage 133: 321-330.

60. Allison, P.D. (2000): Survival analysis using the SAS system. Cary, NC: SAS Institute Inc.

61. Powell TM, and Bagnell ME (2012): Your "survival" guide to using time-dependent covariates. Proceedings of the SAS Global Forum. Available at: https://support.sas.com/resources/papers/proceedings12/168-2012.pdf

62. Allison PD (1995): Survival Analysis Using the SAS System: A Practical Guide. Cary, NC: SAS Institute, Inc. 
63. Cole DM, Beckmann CF, Long CJ, Matthews PM, Durcan MJ, and Beaver JD (2010):

Nicotine replacement in abstinent smokers improves cognitive withdrawal symptoms with modulation of resting brain network dynamics. NeuroImage 52:590-599.

64. Chua HF, Ho SS, Jasinska AJ, Polk TA, Welsh RC, Liberzon I, and Strecher VJ (2011): Selfrelated neural response to tailored smoking-cessation messages predicts quitting. Nat Neurosci 14: 426-427.

65. Cooper N, Tompson S, O’Donnell MB, and Emily BF (2015): Brain activity in self-and value-related regions in response to online antismoking messages predicts behavior change. J Media Psychol 27: 93-109.

66. Falk EB, Berkman ET, Whalen D, and Lieberman MD (2011): Neural activity during health messaging predicts reductions in smoking above and beyond self-report. Health Psychol 30: $177-185$.

67. Newman-Norlund RD, Thrasher JF, Fridriksson J, Brixius W, Froeliger B, Hammond D, and Cummings MK (2014): Neural biomarkers for assessing different types of imagery in pictorial health warning labels for cigarette packaging: a cross-sectional study. BMJ Open 4: e006411.

68. Wang AL, Ruparel K, Loughead JW, Strasser AA, Blady SJ, Lynch KG, et al. (2013):

Content matters: neuroimaging investigation of brain and behavioral impact of televised anti-tobacco public service announcements. J Neurosci 33: 7420-7427.

69. Loughead J, Wileyto EP, Ruparel K, Falcone M, Hopson R, Gur R, and Lerman C (2015). Working memory-related neural activity predicts future smoking relapse. Neuropsychopharmacology 40: 1311-1320. 
70. Booth-Kewley S, Larson GE, and Miyoshi DK (2007): Social desirability effects on computerized and paper-and-pencil questionnaires. Comput Hum Behav 21: 463-477.

71. Nisbett R, and Wilson T (1977): Telling more than we can know: Verbal reports on mental processes. Psychol Rev 84: 231-259.

72. Schlauch RC, Gwynn-Shapiro D, Stasiewicz PR, Molnar DS, and Lang AR (2013): Affect and craving: Positive and negative affect are differentially associated with approach and avoidance inclinations. Addict Behav 38: 1970-1979.

73. Lydon-Staley DM, Cleveland HH, Huhn AS, Cleveland MJ, Harris J, Stankoski D, et al. (2017): Daily sleep quality affects drug craving, partially through indirect associations with positive affect, in patients in treatment for nonmedical use of prescription drugs. Addict Behav 65: 275-282.

74. Zinser MC, Baker TB, Sherman JE, and Cannon DS (1992): Relation between self-reported affect and drug urges and cravings in continuing and withdrawing smokers. $J$ Abnorm Psychol 101: 617-629.

75. Betzel RF, Satterthwaite TD, Gold JI, and Bassett DS (2017): Positive affect, surprise, and fatigue are correlates of network flexibility. Sci Rep 7:1-10.

76. Caplar N, Tacchella S, and Birrer S (2017): Quantitative evaluation of gender bias in astronomical publications from citation counts. Nat Astron 1: 1-5.

77. Chakravartty P, Kuo R, Grubbs V, and McIlwain C (2018): \# CommunicationSoWhite. J Commun 68: 254-266.

78. Dion ML, Sumner JL, and Mitchell SM (2018): Gendered citation patterns across political science and social science methodology fields. Political Anal 26: 312-327. 
79. Dworkin JD, Linn KA, Teich EG, Zurn P, Shinohara RT, and Bassett DS (2020): The extent and drivers of gender imbalance in neuroscience reference lists. arXiv preprint arXiv:2001.01002.

80. Maliniak D, Powers R, and Walter BF (2013): The gender citation gap in international relations. Int Organ 67: 889-922.

81. Thiem Y, Sealey KF, Ferrer AE, Trott AM, and Kennison R (2018): Just Ideas? The Status and Future of Publication Ethics in Philosophy: A White Paper. Technical report.

82. Zhou D, Cornblath EJ, Stiso J, Teich EG, Dworkin JD, Blevins AS, and Bassett DS (2020): Gender diversity statement and code notebook v1.0. Retrieved from https://github.com/dalejn/cleanBib/ 
Table 1

Correlations and Descriptive Statistics of Key Study Variables

\begin{tabular}{|c|c|c|c|c|c|c|c|}
\hline Variables & $\mathbf{1}$ & 2 & 3 & 4 & 5 & 6 & 7 \\
\hline 1. Default Mode Segregationa & - & & & & & & \\
\hline 2. DMN-FPN Connectivity & $-0.76 * * *$ & - & & & & & \\
\hline 3. DMN WN Connectivity ${ }^{a}$ & $0.61 * *$ & 0.04 & - & & & & \\
\hline 4. FPN WN Connectivity ${ }^{\mathrm{a}}$ & -0.24 & $0.54 *$ & 0.32 & - & & & \\
\hline 5. Age & $-0.59 *$ & 0.46 & -0.31 & 0.10 & - & & \\
\hline 6. Cigarettes Per Day & -0.15 & -0.04 & -0.30 & -0.12 & -0.15 & - & \\
\hline 7. Motiona & -0.06 & -0.02 & -0.15 & 0.03 & 0.01 & 0.25 & - \\
\hline Mean & 0.34 & 0.09 & 0.14 & 0.15 & 24.41 & 13.41 & 1.08 \\
\hline Standard Deviation & 0.10 & 0.01 & 0.10 & 0.03 & 6.90 & 3.62 & 0.04 \\
\hline
\end{tabular}

Notes: ${ }^{a}$ Intraindividual mean of the time series (up to 10 scan blocks per person); $N=17 ; * * * p<0.001 ; * * p<0.01 ; * p<0.05$. 
Table 2 .

Cox regression results testing association between default mode frontoparietal system segregation and age on hazard of choosing to leave the scanner to smoke

\begin{tabular}{llllll}
\hline Predictor & Estimate & $\begin{array}{l}\text { Standard } \\
\text { Error }\end{array}$ & $\boldsymbol{p}$ & $\begin{array}{l}\text { Hazard } \\
\text { Ratio }\end{array}$ & $\begin{array}{l}\mathbf{9 5 \%} \\
\text { Confidence } \\
\text { Interval of } \\
\text { Hazard } \\
\text { Ratio }\end{array}$ \\
\hline Default Mode Segregation & -5.84 & 2.82 & 0.04 & 0.003 & $0.00-0.74$ \\
Age & -0.12 & 0.07 & 0.07 & 0.89 & $0.78-1.01$ \\
-2 Log Likelihood & 40.92 & & & & \\
AIC & 46.20 & & & & \\
\hline
\end{tabular}

Note: $\mathrm{AIC}=$ Akaike Information Criteria. $N=17$ persons. Likelihood ratio test: $\chi^{2}(2)=7.02$, $p=0.03$. 


\section{Figure Captions}

Figure 1. Survival times (x-axis) for each participant (separate lines on y-axis). Event times (leaving the scanner to smoke a cigarette) are indicated by an X. Three participants remained in the scanner for the entire 50 minutes. Each scan block for each participant is colored to indicate the magnitude of default mode frontoparietal system segregation. Lighter colors indicate greater segregation.

Figure 2. Estimated baseline survival function. 
Figure 1.

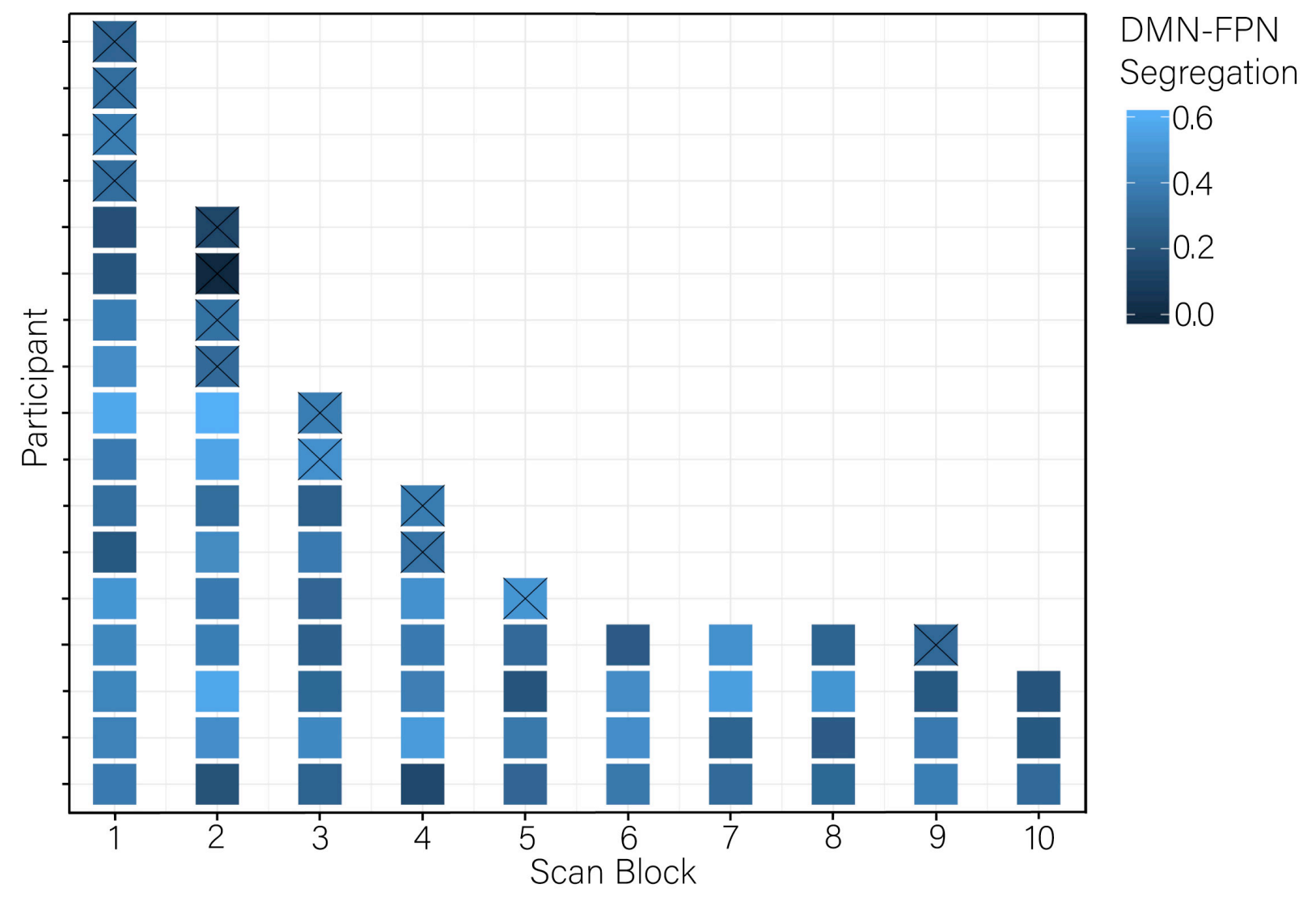


Figure 2.

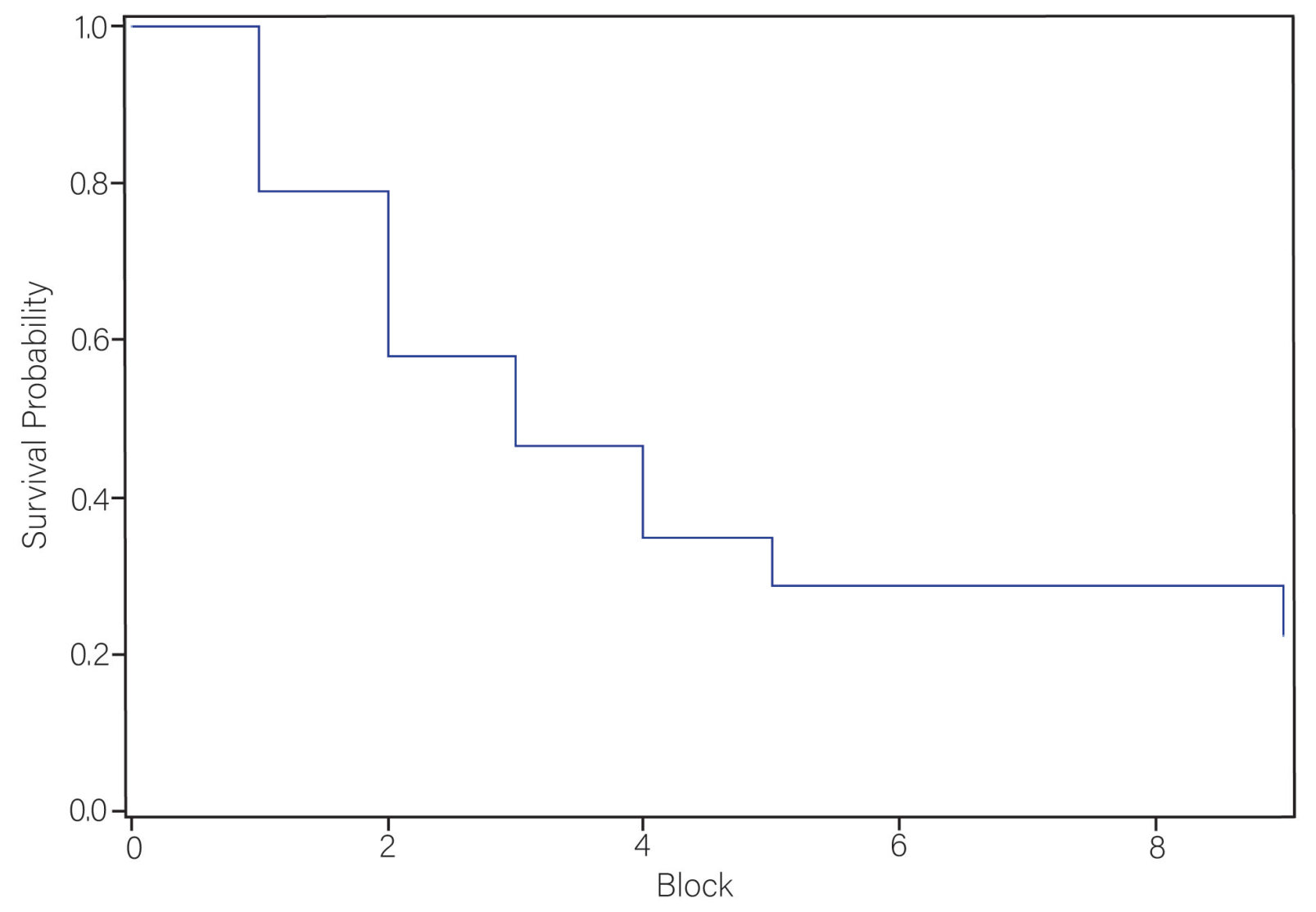

